\title{
Editorial: communication optimization for scalable parallel system
}

\author{
Ching-Hsien Hsu • Peter Sloot
}

Published online: 31 January 2012

(C) Springer Science+Business Media, LLC 2012

Scalable Parallel System has emerged rapidly as an exciting new paradigm that offers a challenging model of computing and poses fascinating problems regarding communication optimizations, ranging from language and algorithm design to compiler optimization and run time system support. This special issue is intended to foster stateof-the-art research in the area of communication optimizations for Scalable Parallel System including the topics of algorithm development, implementation and execution on real-world parallel architecture and novel applications associated with this new paradigm.

The six papers included in this special issue demonstrate the effectiveness and efficiency of a variety of technologies and applications in scalable parallel systems. All of them not only contribute valuable insights and results but also have particular relevance to the parallel computing community. They also present high quality results for tackling problems arising from the ever-growing scalable technologies.

The first paper by Minyi Guo, Weng-Long Chang, Bo Jiang, Shu-Chien Huang, Sien-Tang Tsai and Michael (Shan-Hui) Ho, entitled "Communication-free data alignment for arrays with exponential references in parallelizing compilers for scalable parallel systems" proposes two alignment techniques to properly map, in a communication-free manner, the computations and array references with exponential subscripts or other complex nonlinear subscripts onto the virtual processors. The proposed alignment techniques, which are based on elementary linear algebra, reduce

C.-H. Hsu $(\bowtie)$

Department of Computer Science and Information Engineering, Chung Hua University, Hsinchu, Taiwan

e-mail: chh@chu.edu.tw

P. Sloot

Department of Computer Science, University of Amsterdam, Amsterdam, The Netherlands

e-mail: P.M.A.Sloot@uva.nl 
the alignment problem to the problem of determining a null space basis for a matrix. By simplifying the process of solving the null space basis, the proposed techniques can easily determine the desired mapping functions. The experimental results using SPEC95FP Benchmarks point out that the techniques proposed in the paper can improve the execution time of the subroutines in these benchmarks.

The second paper by Chao-Chin Wu, Lien-Fu Lai, Chao-Tung Yang and Po-Hsun Chiu entitled "Using hybrid MPI and OpenMP programming to optimize communications in parallel loop self-scheduling schemes for multi-core PC clusters" proposes a hybrid MPI and OpenMP programming model for designing two-level parallel loop self-scheduling schemes. In the first level, each computing node runs an MPI process for inter-node communications. In the second level, each processor core runs an OpenMP thread to execute the iterations assigned for its resident node. As a cluster system with multi-core computing nodes is regarded as a two-level hierarchical structure, the first level consists of computing nodes while the second level is comprised of processor cores. In the two-level loop self-scheduling scheme, MPI processes are used for inter-node communications and OpenMP threads are employed for intranode communications. MPI processes communicate with each other by transmitting messages via network, while OpenMP threads communicate with each other by accessing to data in shared memory. The experimental results show that the proposed method delivers significant improvements.

The third paper by Feilong Tang and Minglu Li entitled "Context-adaptive and energy-efficient mobile transaction management in pervasive environments" proposes a context model and a context-aware transaction model and designed mesh network based architecture for online pervasive transactions, and presents a contextadaptive and energy-efficient transaction management mechanism (CETM) that can dynamically adjust transaction execution behaviors in terms of current context information. The proposed transaction processing architecture breakthroughs the limitations of traditional client-proxy-server framework, allowing users to access pervasive services anytime anywhere. The CETM can adapt to dynamical transaction context and reduce energy consumption for a transaction processing. Moreover, the authors model and verify the correctness of the CETM through Petri nets. The simulation results demonstrate that the proposed transaction management mechanism CETM can significantly reduce the failed probability of concurrent pervasive transactions.

In the fourth paper, by Francisco Isidro Massetto Liria Matsumoto Sato and KuanChing Li entitled "A novel strategy for building interoperable MPI environment in heterogeneous high performance systems," a hybrid communication interfacing strategy is proposed, to execute a parallel application in a group of computing nodes belonging to different clusters or multi-clusters (computing systems may be running different operating systems and MPI implementations), interconnected with public or private IP addresses, and responding interchangeably to user execution requests. The authors propose in this paper a hybrid MPI model for integration of several MPI implementations and cluster infrastructures, namely HyMPI. Taking into account the HyMPI development, application developer does not have to rewrite the source code, since HyMPI instruments the same MPI bindings, according to the MPI-2 standard. The only modification needed is to recompile the application, adding the HyMPI 
library into the original application program. Experimental results demonstrate the feasibility of this proposed strategy and its effectiveness, through the execution of benchmarking parallel applications.

The fifth paper by Guillermo L. Taboada, Juan Touriño and Ramón Doallo entitled "F-MPJ: scalable Java message-passing communications on parallel systems" presents a scalable and efficient Message-Passing in Java (MPJ) communication middleware for parallel computing. The increasing interest in Java as the programming language of the multi-core era demands scalable performance on hybrid architectures. However, current Java communication middleware lacks efficient communication support. This work boosts this situation by providing efficient non-blocking communication, which allows communication overlapping and thus scalable performance, taking advantage of shared memory systems and high performance networks through the use of high-performance Java sockets implementation, avoiding the use of communication buffers, and optimizing MPJ collective primitives. The proposed technique significantly improves the scalability of current MPJ implementations. A performance evaluation on an InfiniBand multi-core cluster has shown that the communication primitives outperform representative MPJ libraries up to 60 times.

The last paper by Bibo Tu, Jianping Fan, Jianfeng Zhan and Xiaofang Zhao entitled "Performance analysis and optimization of MPI collective operations on multicore clusters" proposes new parallel computation model to unitedly abstract memory hierarchy on multi-core clusters in vertical and horizontal levels. The new model provides the theoretical underpinning for the optimal design of MPI collective operations. Another contribution of this work is the methodology for optimizing MPI collective operations on multi-core clusters aimed at horizontal memory hierarchy. The optimal implementation of a collective for a given system mainly depends on virtual topology (e.g. flat-tree, binary tree, binomial tree, etc.) and message sizes. Experimental results show that the new model can predict communication costs for message passing on multi-core clusters more accurately than the previous models.

All of the above papers address either technical issues in scalable and parallel computing systems or propose novel application models in the various scalable computing fields. They also trigger further related research and technology improvements in application and services of scalable systems. Honorably, this special issue serves as a landmark source for education, information, and reference to professors, researchers and graduate students interested in updating their knowledge about, or being active in, application models for scalable and parallel computing and communication systems.

The guest editor would like to express sincere gratitude to Prof. Hamid R. Arabnia, the Editor-in-Chief of the Journal of Supercomputing, for giving us the opportunity to prepare this special issue. In addition, I am deeply indebted to numerous reviewers for their professional effort, insight and hard work put into commenting on the selected articles which reflect the essence of this special issue. Last but not least, I am grateful to all the authors for their contributions and for undertaking two-cycle revision of their manuscripts, without which this special section could not have been produced.

Finally, we hope the readers will enjoy these selected papers as we did and will find this issue informative and helpful in keeping themselves updated in the fast changing field of the "Scalable Computing Era." 